\title{
Computer-assisted ultrasound assessment of plaque characteristics in radiation-induced and non-radiation-induced carotid atherosclerosis
}

\author{
Yuanxi $\mathrm{Li}^{1 \wedge}$, Dora Lai-Wan Kwong ${ }^{2} \wedge$, Vincent Wing-Cheung $\mathrm{Wu}^{1} \wedge$, Shea-Ping $\mathrm{Yip}^{1 \wedge}$, \\ Helen Ka-Wai Law ${ }^{1} \wedge$, Shara Wee-Yee Lee ${ }^{1 \wedge}$, Michael Tin-Cheung Ying ${ }^{1 \wedge}$ \\ ${ }^{1}$ Department of Health Technology and Informatics, The Hong Kong Polytechnic University, Hong Kong, China; ${ }^{2}$ Department of Clinical \\ Oncology, The University of Hong Kong, Queen Mary Hospital, Hong Kong, China
}

Correspondence to: Dr. Michael Tin-Cheung Ying, PhD. Department of Health Technology and Informatics, The Hong Kong Polytechnic University, Hung Hom, Kowloon, Hong Kong, China. Email: michael.ying@polyu.edu.hk.

\begin{abstract}
Background: This study investigated the feasibility of using a computer-assisted method to evaluate and differentiate the carotid plaque characteristics in radiation-induced and non-radiation-induced carotid atherosclerosis.

Methods: This study included 107 post-radiotherapy (post-RT) nasopharyngeal carcinoma (NPC) patients and 110 subjects with cardiovascular risk factors (CVRFs). Each participant had a carotid ultrasound examination, and carotid plaques and carotid intima-media thickness (CIMT) were evaluated with grey scale ultrasound. The carotid plaque characteristics were evaluated for grey-scale median (GSM) and detailed plaque texture analysis (DPTA) using specific computer software. In DPTA, five different intra-plaque components were colour-coded according to different grey scale ranges. A multivariate linear regression model was used to evaluate the correlation of risk factors and carotid plaque characteristics.
\end{abstract}

Results: Post-RT NPC patients have significantly higher CIMT $(748 \pm 15.1 \mu \mathrm{m}, \mathrm{P}=0.001)$, more patients had a plaque formation $(80.4 \%, \mathrm{P}<0.001)$ and more plaque locations $(2.3 \pm 0.2, \mathrm{P}<0.001)$ than CVRF subjects $(680.4 \pm 10.0 \mu \mathrm{m}, 38.2 \%$ and $0.5 \pm 0.1$ respectively). Among the five intra-plaque components, radiationinduced carotid plaques had significantly larger area of calcification $(4.8 \% \pm 7.7 \%, \mathrm{P}=0.012)$, but lesser area of lipid $(42.1 \% \pm 16.9 \%, \mathrm{P}=0.034)$ when compared to non-radiation-induced carotid plaques $(3.0 \% \pm 5.7 \%$ and $46.3 \% \pm 17.9 \%$ respectively). Age, radiation and number of CVRF were significantly associated with the carotid atherosclerosis burden $(\mathrm{P}<0.001)$. Besides, age was significantly associated with the amount of lipid and calcification within carotid plaques $(\mathrm{P}<0.001)$.

Conclusions: Radiation caused more severe carotid artery disease than CVRF with larger CIMT and more prevalent of carotid plaque. Radiation-induced carotid plaques tended to have more intra-plaque calcifications, whereas non-radiation-induced carotid plaques had more lipids. Ultrasound aided by computer-assisted image analysis has potential for more accurate assessment of carotid atherosclerosis.

Keywords: Atherosclerotic plaque; carotid atherosclerosis; radiation effects; ultrasound

Submitted Aug 29, 2020. Accepted for publication Jan 07, 2021.

doi: $10.21037 /$ qims-20-1012

View this article at: http://dx.doi.org/10.21037/qims-20-1012

\footnotetext{
$\wedge$ ORCID: Yuanxi Li, 0000-0002-9309-5621; Dora Lai-Wan Kwong, 0000-0002-1304-2854; Vincent Wing-Cheung Wu, 0000-00021257-4154; Shea-Ping Yip, 0000-0002-2170-8185; Helen Ka-Wai Law, 0000-0002-5579-9054; Shara Wee-Yee Lee, 0000-0001-5257-2076; Michael Tin-Cheung Ying, 0000-0001-5979-6072.
} 


\section{Introduction}

Nasopharyngeal carcinoma (NPC) is a common head and neck cancer in Southeast Asia. In 2018, there were 129,079 new NPC cases globally, and $76.7 \%$ of the cases were in Asia, of which 26.9\% were in Southeast Asia (1). Nodal metastasis is common in NPC patients, and about $60 \%$ to $85 \%$ of the patients present neck lymph node metastasis (2). For NPC, radiotherapy (RT) is the common treatment method for both the primary tumour and metastatic neck lymph nodes, and chemotherapy may be used in conjunction with RT for patients in advanced stages of the disease (3). Since extracranial carotid arteries are in close proximity to neck lymph nodes, they are unavoidably irradiated during the RT of neck lymph nodes $(4,5)$.

Carotid atherosclerosis is a late post-RT complication in patients who have received external irradiation to the head and neck. As a chronic inflammatory disease, subclinical vascular disease is identified by the increased carotid intima-media thickness (CIMT) and the formation of carotid atherosclerotic plaque (6-8). The development of carotid atherosclerosis is a progressive process, and therefore different characteristics of carotid plaques represent different stages of atherosclerotic development. It is generally believed that atherosclerosis is initiated by endothelial dysfunction on the inner surface of artery wall. The injured endothelium elicits the sub-endothelial accumulation of cholesterol, namely oxidized low-density lipoprotein (LDL) (9-11).

The composition of carotid plaque is largely associated with the risk of stroke of patients. Advanced atherosclerotic lesion forms a thrombus, which can occlude the blood vessel. Rupture and erosion of carotid plaque may form emboli in the circulation which lead to stroke and cerebrovascular events. Therefore, carotid atherosclerosis and the plaque formation increase the risk of cerebrovascular diseases $(8,10,12,13)$.

Clinically, ultrasound is a non-invasive imaging tool and is commonly used to evaluate carotid artery disease by examining various parameters including CIMT, carotid arterial stiffness and carotid plaque score. Nevertheless, the difference of radiation-induced and non-radiation-induced carotid atherosclerosis was not comprehensively investigated. Computer-aided assessment of carotid plaque characteristics on ultrasound images is recently available $(14,15)$. However, there is no previous studies used this method to assess radiation-induced and non-radiation-induced carotid plaques, and compare their differences.
In the present study, we aimed to evaluate the degree of carotid atherosclerosis of NPC patients with previous RT and subjects with cardiovascular risk factor (CVRF) by evaluating their CIMT and carotid plaque formation using ultrasonography. This study also investigated the differences in carotid plaque characteristics in radiation-induced and non-radiation-induced carotid atherosclerosis by using a computer-assisted ultrasound assessment method.

\section{Methods}

\section{Subject recruitment}

Post-RT NPC patients treated with intensity-modulated radiation therapy were recruited in the Department of Clinical Oncology of Queen Mary Hospital during follow up. The inclusion criteria of post-RT NPC patients were Chinese NPC patients, 18 years old or above, a single completed RT course for the primary tumour and neck lymph nodes, and a post-RT duration of 4 years or more. The exclusion criteria were a known history of leukopenia, thrombocytopenia, severe hepatic or renal dysfunction, an evidence for inflammatory or other malignant diseases, and a known history of carotid atherosclerosis prior to RT, previous carotid endarterectomy or stenting.

Subjects with CVRF were recruited by poster advertisement in the Hong Kong Polytechnic University. The inclusion criteria of CVRF subjects were Chinese, older than 40 years, and at least having one CVRF, including smoking (current smoker), diabetes mellitus (DM), hypertension, hypercholesterolemia or coronary heart disease. The criteria for identifying these CVRFs were the same as described in our previous study (16). The exclusion criteria were a known history of leukopenia, thrombocytopenia, severe hepatic or renal dysfunction, an evidence for inflammatory or other malignant diseases, and a known history of carotid atherosclerosis and underwent RT, carotid endarterectomy or stenting previously.

\section{Study design and ultrasound examination}

The Human Subject Ethics Subcommittee of the Hong Kong Polytechnic University (HSEARS20160930001) and the Institutional Review Board of the University of Hong Kong/Hospital Authority Hong Kong West Cluster approved this study (HA RE001F3). All participants (recruited post-RT NPC patients and CVRF subjects) were provided an information sheet with detailed information of 
the study and the rights of participants. Informed written consent was obtained from all participants before the commencement of the examinations.

All recruited participants had a carotid ultrasound examination. All ultrasound examinations were performed by a single dedicated ultrasound researcher with 5 years of carotid ultrasound experience using the Esaote $\mathrm{MyLab}^{\mathrm{TM}}$ Twice eHD CrystaLine ultrasound unit in conjunction with a 3-13 MHz linear transducer (Esaote, Genoa, Italy). In order to ensure measurements were obtained with the subjects at resting state, all subjects were allowed to rest for at least 10 minutes before the ultrasound examination. In the carotid ultrasound examination, the subject lay supine on the examination couch with the shoulders and neck supported by a pillow so that the neck was slightly extended and the head turned away from the side under examination. On each side of the neck, the CIMT was evaluated using the automated quantification programs of the ultrasound unit: radiofrequency-based quality intima-media thickness (RF-QIMT).

For each subject, the presence or absence of carotid plaque in the internal, external and common carotid arteries was assessed. Carotid plaque was identified as focal arterial wall thickening $>50 \%$ of the adjacent intima-media layer (17). In any cases that the carotid plaque was not clearly demonstrated on grey scale ultrasound such as hypoechoic plaque, colour Doppler ultrasound was used to demonstrate the vessel lumen at the stenosis for identify the carotid plaque.

In the evaluation of grey-scale median (GSM) and detailed plaque texture analysis (DPTA), archived digital images were reviewed and analysed using image-processing software Adobe Photoshop CS v.8.0 (Adobe, San Jose, CA, United States) and Image Pro Plus v.6.0 (Media Cybernetics, Rockville, MD, United States). For each carotid plaque, five longitudinal sonograms which clearly demonstrating the borders and internal echotexture of the plaque were selected to evaluate the GSM and DPTA. Image normalization was performed before the evaluations. With the use of the histogram facility of the software, the grey scale value of two reference areas (blood and adventitia) in the image were adjusted to standardise the grey scale value of blood as 0 and adventitia as 190. All the pixels of the image were adjusted accordingly based on the standard linear scale, and a normalized image was produced (18-20).

For the evaluation of GSM, the carotid plaque was outlined manually on the normalized grey scale ultrasound image using software Adobe Photoshop CS, v.8.0 (Adobe, San Jose, CA, United States) using the function "Lasso
Tool". The software then calculated the median of the grey scale value of pixels (i.e., GSM) within the region of interest (ROI) and the GSM value was displayed in the Histogram window.

For the DPTA, the software Image Pro Plus v.6.0 (Media Cybernetics, Rockville, MD, United States) was used to analyse the normalized grey scale ultrasound images. In the image analysis, the different components of carotid plaque were color-coded by the software based on the different grey scale ranges as suggested by a previous literature $(14,15)$ : yellow for blood (grey scale ranges, 0-9), orange for lipid [10-31], red for muscle [32-74], light blue for fibrous tissue [75-111] and dark blue for calcium [112-255]. On ultrasound, the blood, lipid and muscle appeared hypoechoic, while fibrous tissue and calcification were hyperechoic [75255] (15). Each carotid plaque component was evaluated by three parameters, namely average pixel density, area percentage and integrated optical density (IOD). Average pixel density (or intensity) indicated the mean value of pixel densities of each component. Area percentage indicated the percentage that the area of each component relative to the area of the entire plaque. IOD indicated integration of pixel density of each component, which was also equal to area $x$ average pixel density. Thus, it was an accumulative factor account for both pixel density and area percentage of each component.

\section{Data analysis}

Basic information of the subjects and plaque characteristics were expressed as mean \pm SD or SEM for the continuous data; counts and percentage were presented for categorical data. Normal distribution of the data was evaluated by Shapiro-Wilk test. The difference between post-RT NPC group and CVRF group were evaluated using Mann Whitney $U$ test and Chi-square test for nonparametric variables (number of CVRFs, plaque number per subject, plaque formation, CIMT and GSM), and unpaired Student $t$-test for parametric variables (age). Comparisons among groups were evaluated by one-way ANOVA. The effects of age, sex, number of CVRFs and exposure to radiation on carotid plaque characteristics were analysed by multiple linear regression models. The risk factors regarded as candidate variables were age, sex, number of CVRFs and radiation. We used multivariate linear regression model to analyse the effects of different risk factors on plaque characteristics (CIMT, plaque formation, number of plaques per subject, plaque components). All statistical analyses 
Table 1 Basic information of the study subjects

\begin{tabular}{|c|c|c|c|}
\hline Parameters & Post-RT NPC $(n=107)$ & CVRF $(n=110)$ & $P$ value \\
\hline Sex (female/male), $\mathrm{n}$ & $39 / 68$ & $66 / 44$ & $<0.001^{\star \star \star}$ \\
\hline Chemotherapy & $59(55.1)$ & - & - \\
\hline Post-RT duration & $15 \pm 8.9$ & - & - \\
\hline 0 & 57 (53.3) & - & - \\
\hline 1 & $31(28.9)$ & $58(52.7)$ & $<0.001^{\star \star \star}$ \\
\hline$\geq 2$ & $19(17.8)$ & $52(47.3)$ & $<0.001^{\star * *}$ \\
\hline \multicolumn{4}{|l|}{ CVRF } \\
\hline Diabetes & $6(5.6)$ & $23(20.9)$ & $<0.001^{\star \star \star}$ \\
\hline Current smoking & $6(5.6)$ & $9(8.2)$ & 0.455 \\
\hline Heart disease & $4(3.7)$ & - & $0.041^{*}$ \\
\hline
\end{tabular}

Values are expressed as mean $\pm \mathrm{SD}$ or $\mathrm{n}(\%) .{ }^{*}, \mathrm{P}<0.05 ;{ }^{* \star}, \mathrm{P}<0.001 . \mathrm{RT}$, radiotherapy; NPC, nasopharyngeal carcinoma; CVRF, cardiovascular risk factor.

were performed using SPSS 22 (IBM Corporation, Armonk, NY, United States) and Prism 7.0 (GraphPad Software, San Diego, CA, United States). P value $<0.05$ was considered to be significant.

\section{Results}

\section{Demographic information}

From April 2017 to May 2018, a total of 111 post-RT NPC patients and $110 \mathrm{CVRF}$ subjects were recruited. Four postRT patients were excluded from the study because two of them did not have comprehensive clinical information, and two were unsatisfied to the inclusion criteria, because the post-RT duration of one patient was less than 4 years, and another patient was treated with stenting without informing the researcher during the face-to-face interview. Finally, 107 post-RT NPC patients and 110 CVRF subjects were included in the study. The demographic and clinical information of the post-RT NPC patients and CVRF subjects are summarized in Table 1 . There was no significant difference in the age of the post-RT NPC patients and CVRF subjects $(58.4 \pm 1.1$ vs. $60.7 \pm 0.7$ respectively, $\mathrm{P}>0.05)$. There were significantly more males among the post-RT
NPC patients when compared to the CVRF group (68/107 vs. 44/110, $\mathrm{P}<0.001)$. Among the post-RT NPC patients, 57 of them (53.3\%) had no CVRFs, 31 patients (28.9\%) had one CVRF and 19 patients $(17.8 \%)$ had two or more CVRFs.

In the CVRF group, 58 out of 110 subjects (52.7\%) had only one CVRF and the remaining subjects had at least two CVRFs (47.3\%). The most prevalent CVRF was hypertension $(\mathrm{n}=74,67.3 \%)$, followed by dyslipidemia $(\mathrm{n}=69,62.7 \%)$, diabetes $(\mathrm{n}=23,20.9 \%)$ and current smoker $(\mathrm{n}=9,8.2 \%)$.

\section{Carotid plaque assessment}

Table 2 shows the comparison of the characteristics of radiation-induced and non-radiation-induced carotid atherosclerosis. Results showed that post-RT NPC patients were more likely to have carotid plaques when compared to the CVRF subjects $(80.4 \%$ vs. $38.2 \%, \mathrm{P}<0.001)$. Regardless of the presence or absence of CVRF, the mean number of carotid plaques in post-RT NPC patients was significantly higher than that in CVRF subjects $(\mathrm{P}<0.001)$. There was no significant difference in the number of carotid plaques between post-RT NPC patients with or without CVRF 

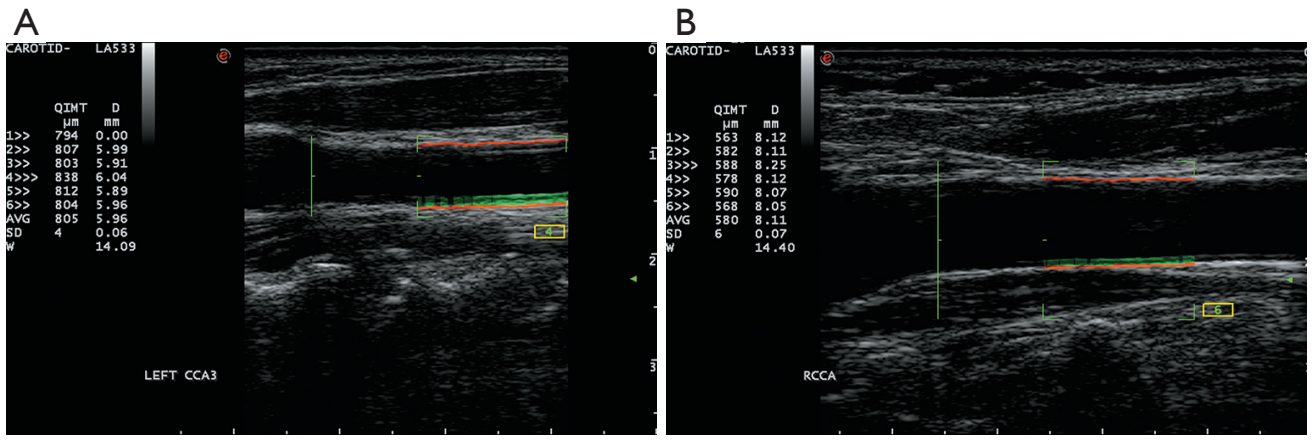

Figure 1 The comparison of CIMT between post-RT NPC and CVRF subjects. Longitudinal sonograms show CIMT of 1-cm segment in $1 \mathrm{~cm}$ proximal to the inferior end of the carotid bulb of a post-RT NPC patient (A) and of a CVRF subject (B). Noted the radiationinduced CIMT is higher (A) than the non-radiation-induced one (B). CIMT, carotid intima-media thickness; RT, radiotherapy; NPC, nasopharyngeal carcinoma; CVRF, cardiovascular risk factor.

Table 2 Comparison of the characteristics between radiation-induced and non-radiation-induced carotid atherosclerosis

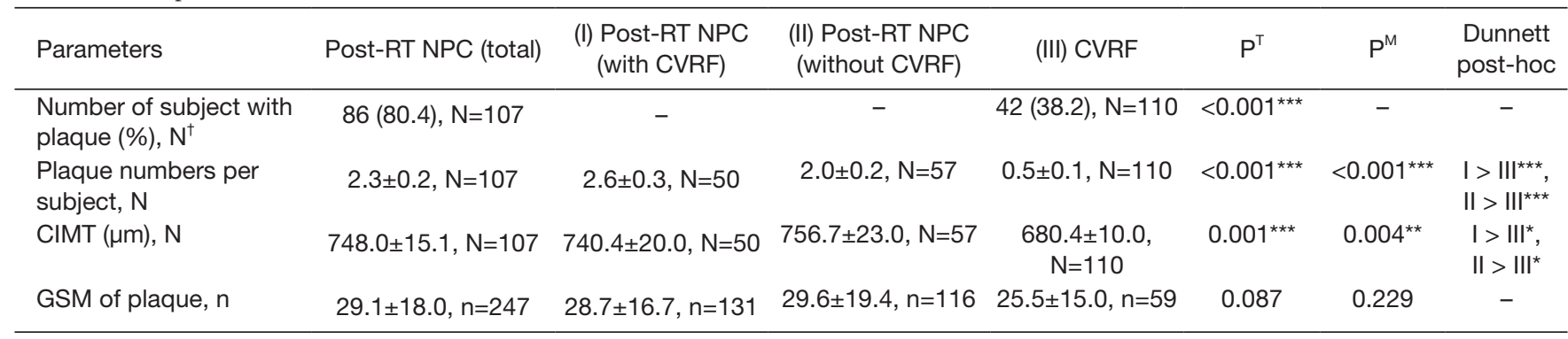

Values are mean \pm SEM. ${ }^{\dagger}, \mathrm{N}=$ total number of subject in the corresponding analysis; $n=$ total number of carotid plaque in the corresponding analysis; $\mathrm{P}^{\top}=$ comparison between total post-RT NPC patients and CVRF subjects; $\mathrm{P}^{\mathrm{M}}=$ multiple comparison among postRT NPC patients with CVRF, post-RT NPC patients without CVRF, and CVRF subjects. ${ }^{*}, \mathrm{P}<0.05$; ${ }^{* *}, \mathrm{P}<0.01$; ${ }^{* \star}, \mathrm{P}<0.001$. RT, radiotherapy; NPC, nasopharyngeal carcinoma; CVRF, cardiovascular risk factor; CIMT, carotid intima-media thickness; GSM, grey-scale median.

$(\mathrm{P}>0.05)$. Among the 247 carotid plaques in the post-RT NPC patients, 7 (2.8\%) were located in internal carotid artery (ICA), 160 (64.8\%) were in carotid bulb, and 80 $(32.4 \%)$ were in common carotid artery (CCA). Among the 59 carotid plaques in the CVRF subjects, 3 (5.1\%) were found in ICA, 2 (3.4\%) were in external carotid artery, 45 $(76.3 \%)$ were in carotid bulb, and $9(15.2 \%)$ in CCA.

\section{CIMT assessment}

As shown in the Figure 1 and Table 2, post-RT NPC patients had significantly larger CIMT $(748 \pm 15.1 \mu \mathrm{m})$ than CVRF subjects $(680.4 \pm 10 \mu \mathrm{m}, \mathrm{P}=0.001)$. Besides, both post-RT NPC patients with CVRF $(740.4 \pm 20 \mu \mathrm{m})$ and those without CVRF $(756.7 \pm 23 \mu \mathrm{m})$ had significantly larger CIMT than subjects in CVRF group $(680.4 \pm 10 \mu \mathrm{m}, \mathrm{P}=0.004)$. However, the difference in the CIMT between post-RT NPC patients with CVRF and those without CVRF was not statistically significant $(\mathrm{P}>0.05)$.

\section{GSM assessment}

Results showed that there was no significant difference in the GSM of carotid plaque in post-RT NPC patients and CVRF subjects $(\mathrm{P}=0.087)$, nor among post-RT NPC patients with or without CVRF and CVRF subjects $(\mathrm{P}=0.229)$ (Table 2).

\section{Carotid plaque component assessment}

Tables 3,4 show the DPTA results in terms of the presence of different components within the carotid plaque of post- 
Table 3 Comparison of the presence of different components within the carotid plaques in post-RT NPC patients and CVRF subjects

\begin{tabular}{lccc}
\hline \multirow{2}{*}{ Components } & \multicolumn{2}{c}{ Number of carotid plaques $(\%)$} & \multirow{2}{*}{ P value $^{\dagger}$} \\
\cline { 2 - 3 } Blood & Post-RT NPC $(\mathrm{n}=247)$ & $55(93.2)$ & 0.885 \\
Lipid & $229(92.7)$ & $59(100.0)$ & 0.625 \\
Muscle & $246(99.6)$ & $59(100.0)$ & $>1.000$ \\
Fibrous tissue & $247(100.0)$ & $52(88.1)$ & 0.159 \\
Calcification & $231(93.5)$ & $34(57.6)$ & $0.015^{\star}$ \\
\hline
\end{tabular}

${ }^{\dagger}, \mathrm{P}$ value $=$ difference between post-RT NPC patients and CVRF subjects. ${ }^{*}, \mathrm{P}<0.05 . \mathrm{RT}$, radiotherapy; NPC, nasopharyngeal carcinoma; CVRF, cardiovascular risk factor.

Table 4 Comparison of the presence of different components within the carotid plaques among post-RT NPC patients (with or without CVRF) and CVRF subjects

\begin{tabular}{|c|c|c|c|c|}
\hline Components & \multicolumn{3}{|c|}{ Number of plaques (\%) } & $P$ value \\
\hline Blood & $112(93.1)$ & $107(81.7)$ & 55 (93.2) & 0.955 \\
\hline Lipid & $131(100.0)$ & $115(99.1)$ & $59(100.0)$ & 0.379 \\
\hline Muscle & $131(100.0)$ & $116(100.0)$ & $59(100.0)$ & - \\
\hline Calcification & $99(75.6)$ & $83(71.6)$ & $34(57.6)$ & $0.041^{*}$ \\
\hline
\end{tabular}

${ }^{\dagger}, \mathrm{P}$ value $=$ difference in post-RT NPC patients (with/without CVRF) and CVRF subjects. * $\mathrm{P}<0.05 . \mathrm{RT}$, radiotherapy; NPC, nasopharyngeal carcinoma; CVRF, cardiovascular risk factor.

RT NPC patients and CVRF subjects. Results showed that carotid plaques in post-RT NPC patients, regardless the patients had CVRF or not, were more often to have calcification (total, 73.7\%; with CVRF, 75.6\%; without CVRF, 71.6\%) when compared to carotid plaques in CVRF subjects $(57.6 \%)$, and the differences were statistically significant $(\mathrm{P}=0.015, \mathrm{P}=0.041$, respectively). By contrast, no significant differences were observed in the other four plaque components (blood, lipid, muscle and fibrous tissue) among the study groups $(\mathrm{P}>0.05)$.

Carotid plaque components were further evaluated for average pixel density, area percentage and IOD. Table 5 shows the differences of these plaque component parameters between post-RT NPC patients and CVRF subjects. Table 6 compares the plaque component parameters among the postRT NPC patients with or without CVRF and CVRF subjects. There was no significant difference in the average pixel density of the five plaque components between post-RT NPC and CVRF groups $(\mathrm{P}>0.05)$.

For the area percentage of different components within carotid plaque, CVRF subjects had significantly larger area of lipid $(46.3 \% \pm 17.9 \%)$ than all the post-RT NPC patients $(42.1 \% \pm 16.9 \%, \mathrm{P}=0.034$, Table 5, Figure 2$)$ and post-RT NPC patients with CVRF $(40.5 \% \pm 16.1 \% ; \mathrm{P}=0.020$, Table 6). Conversely, carotid plaques in CVRF subjects had significantly lesser calcification $(3.0 \% \pm 5.7 \%)$ than post-RT NPC patients $(4.8 \% \pm 7.7 \%, \mathrm{P}=0.012$, Table 5 , Figure 2$)$ and post-RT NPC patients with CVRF $(4.4 \% \pm 6.6 \%, \mathrm{P}=0.030$, Table 6). No significant differences were found between post-RT NPC patients and CVRF subjects in the area percentage of blood, muscle and fibrous tissue within carotid plaque $(\mathrm{P}>0.05)$.

For the IOD of carotid plaque components, the aggregate post-RT NPC patients had a significantly higher value than CVRF subjects in both muscle $(3,086.0 \pm 3,121.0$ and 2,392.0 $\pm 1,911.0$ respectively, $\mathrm{P}=0.043)$ and calcification $(4,073.0 \pm 6,667.0$ and 2,675.0 $\pm 6,615.0$ respectively, $\mathrm{P}=0.007)$ (Table 5). Carotid plaques in post-RT NPC patients with CVRF had significant higher IOD of muscle $(3,416.0 \pm 3,106.0)$ when compared to those in post-RT NPC patients without CVRF $(2,712 \pm 3,108)$ and CVRF 
Table 5 Difference of carotid plaque component parameters between post-RT NPC patients and CVRF subjects

\begin{tabular}{|c|c|c|c|}
\hline Parameters & Post-RT NPC ( $\mathrm{n}=247)$ & CVRF $(n=59)$ & Pvalue $^{\dagger}$ \\
\hline Blood & $7.5 \pm 2.9$ & $7.3 \pm 1.0$ & 0.316 \\
\hline Lipid & $22.1 \pm 3.7$ & $21.4 \pm 4.5$ & 0.432 \\
\hline Muscle & $44.6 \pm 6.4$ & $43.2 \pm 6.4$ & 0.072 \\
\hline Calcification & $129.5 \pm 11.8$ & $130.2 \pm 13.36$ & 0.851 \\
\hline \multicolumn{4}{|c|}{ Area percentage (\%) } \\
\hline Blood & $14.8 \pm 14.1$ & $15.0 \pm 13.4$ & 0.720 \\
\hline Lipid & $42.1 \pm 16.9$ & $46.3 \pm 17.9$ & $0.034^{*}$ \\
\hline Calcification & $4.8 \pm 7.7$ & $3.0 \pm 5.7$ & $0.012^{*}$ \\
\hline \multicolumn{4}{|l|}{ IOD } \\
\hline Blood & $224.4 \pm 938.0$ & $143.3 \pm 197.4$ & 0.802 \\
\hline Lipid & $1,986.0 \pm 2,157.0$ & $2,056.0 \pm 1,967.0$ & 0.498 \\
\hline Muscle & $3,086.0 \pm 3,121.0$ & $2,392.0 \pm 1,911.0$ & $0.043^{*}$ \\
\hline Fibrous tissue & $1,147.0 \pm 1,112.0$ & $1,090.0 \pm 1,181.0$ & 0.278 \\
\hline Calcification & $4,073.0 \pm 6,667.0$ & $2,675.0 \pm 6,615.0$ & $0.007^{\star \star}$ \\
\hline
\end{tabular}

Values are presented as mean $\pm \mathrm{SD} .{ }^{\dagger}, \mathrm{P}$ value $=$ difference between total post-RT NPC patients and CVRF subjects. ${ }^{*}, \mathrm{P}<0.05 ;{ }^{* *}, \mathrm{P}<0.01$. $\mathrm{RT}$, radiotherapy; NPC, nasopharyngeal carcinoma; CVRF, cardiovascular risk factor; IOD, integrated optical density.

subjects $(2,392.0 \pm 1,911.0)(\mathrm{P}=0.004)$ (Table 6). Additionally, carotid plaques in post-RT NPC patients with CVRF had significant higher IOD of calcification $(3,767.0 \pm 5,593.0)$ when compared to carotid plaques in CVRF subjects $(2,675.0 \pm 6,615.0)(\mathrm{P}=0.018)$ (Table 6).

\section{Association of carotid atherosclerosis parameters and various risk factors in post-RT NPC}

Carotid atherosclerosis parameters that showed significant difference between post-RT NPC patients and CVRF subjects in the previous analyses were further evaluated for their associations with different risk factors. Table 7 summarises the effects of various risk factors on atherosclerosis related parameters in post-RT NPC patients. The patients were divided into three subgroups: (I) without CVRF, (II) with one CVRF, and (III) with more than one CVRFs. After adjustment of age and sex, results showed that age and radiation had significantly impact on the CIMT, the plaque formation and number of carotid plaque $(\mathrm{P} \leq 0.001)$. The number of $C V R F$ was significantly associated with CIMT and number of carotid plaque $(\mathrm{P}<0.05)$. The elder subjects tended to have a higher CIMT [r $=0.36$; $95 \%$ CI: $(0.23$ to 0.47$)]$ and larger number of plaques [ $\mathrm{r}=0.25 ; 95 \% \mathrm{CI}$ : (0.12 to 0.37$)]$.

Among the risk factors studied, age played a significant role in the area percentage of lipid and calcification within a plaque $(\mathrm{P}<0.001$, Table 8$)$. Results show that advancing age of patient decrease the area percentage of intra-plaque lipid [ $\mathrm{r}=-0.27 ; 95 \%$ CI: $(-0.43$ to -0.10$)]$, but increases the area percentage of calcification within carotid plaque [r=-0.29; $95 \%$ CI: (0.12 to 0.44)]. Additionally, radiation has significant influence on the IOD of intra-plaque muscle component $(\mathrm{P}=0.046)$ in a positive correlation pattern $(r=0.116)$. However, results indicated that none of the risk factors played statistically significant role on the IOD of 
Table 6 Differences of carotid plaque component parameters among post-RT NPC patients (with or without CVRF) and CVRF subjects

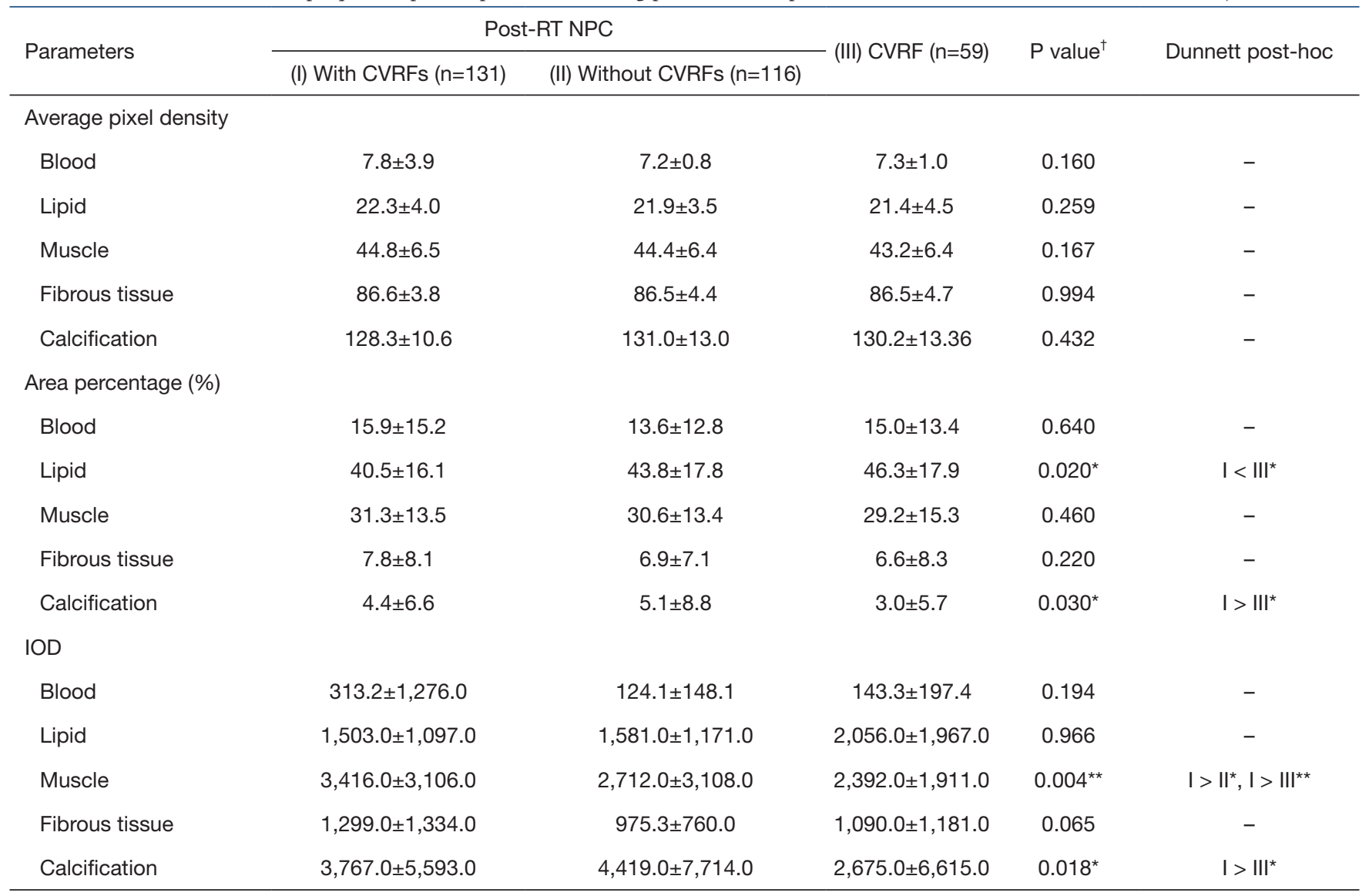

Values are presented as mean $\pm \mathrm{SD} .^{\dagger}, \mathrm{P}$ value $=$ difference among post-RT NPC patients with/without CVRF vs. CVRF subjects. ${ }^{*}, \mathrm{P}<0.05$;

**, P<0.01. RT, radiotherapy; NPC, nasopharyngeal carcinoma; CVRF, cardiovascular risk factor; IOD, integrated optical density.

calcification (Table 8).

\section{Discussion}

In this study, we investigated the difference in CIMT and carotid plaque components in post-RT NPC patients and CVRF subjects respectively. For unveiling the plaque components characteristics, we used computer-assisted methods to evaluate the plaque GSM (21) and perform DPTA (14). Besides, we also investigated the effects of common risk factors on plaque constituents. The major findings of the present study are as follows: (I) radiation leads to higher CIMT and likelihood to develop carotid plaques; (II) there is no significant difference in the GSM between radiation-induced and non-radiation-induced carotid plaques; (III) DPTA results indicated that radiation-induced carotid plaques tend to have more calcification, while non-radiationinduced carotid plaques have more lipid contents, and thus are more unstable; (IV) the plaque components are affected by the age of patient and exposure to radiation.

\section{Carotid intima-media thickness}

In this study, we examined CIMT at the far wall of CCA and with the ultrasound beam perpendicular to the CCA. This scanning method allowed accurate measurement and had high measurement reliability (22-24).

The difference in radiation-induced atherosclerosis and spontaneous atherosclerosis was rarely studied. A previous study reported that there was no significantly difference in CIMT between post-RT NPC patients and diabetes patients $\left(\mathrm{P}_{\mathrm{cor}}=0.732\right)$ (13). The study also found that the males and elderly tended to have higher CIMT (13). However, our study showed that post-RT NPC patients, regardless of the presence of CVRF, had a higher CIMT than CVRF subjects. This result was consistent with the 

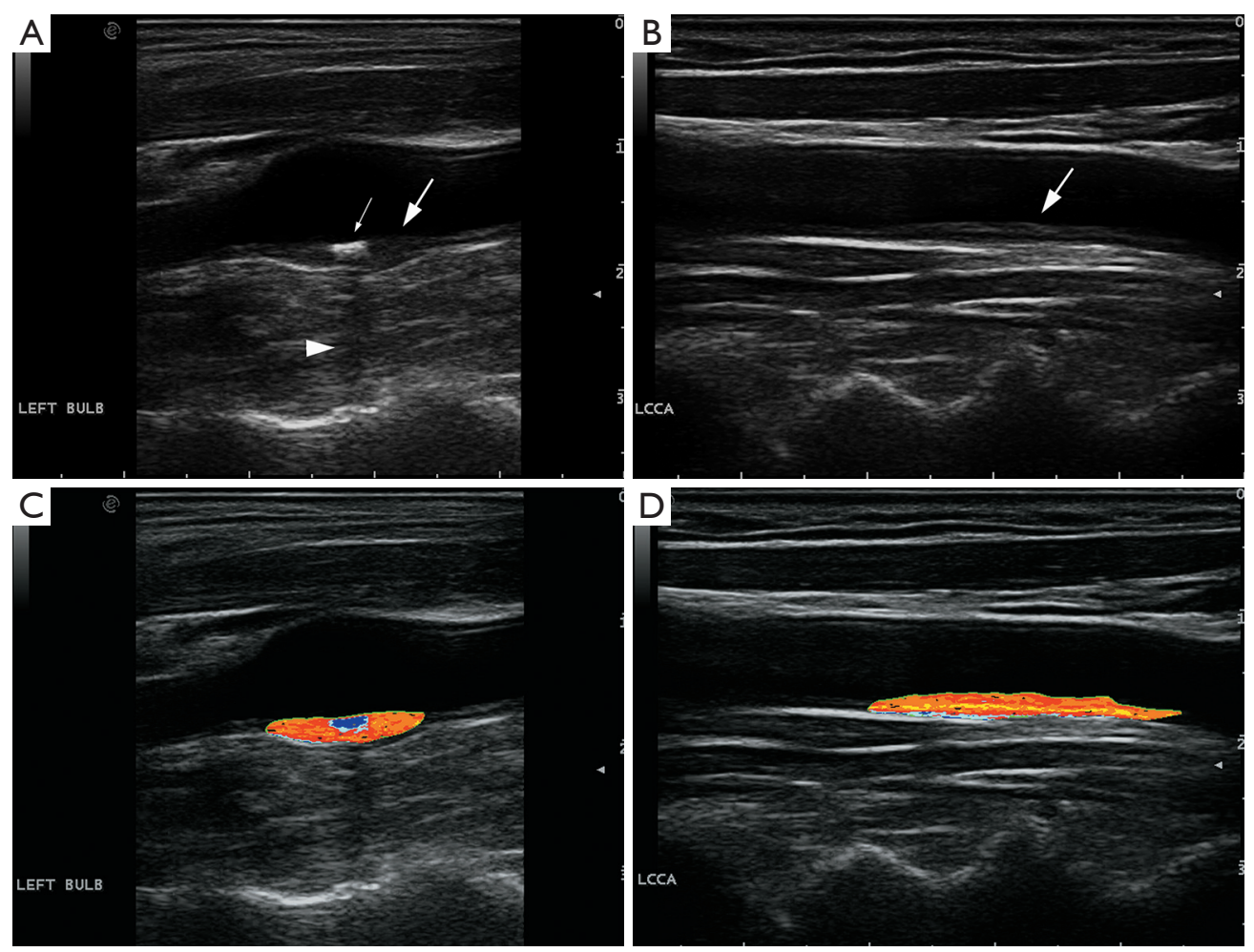

Figure 2 Grey scale ultrasound and DPTA of carotid plaque. Longitudinal sonograms show carotid plaques (large arrows) in the carotid bifurcation of a post-RT NPC patient (A) and in the CCA of a CVRF subject (B). In image (A), note the intra-plaque calcification that appears hyperechoic (small arrow) with acoustic shadowing (arrowhead). Images (C,D) show the detailed plaque texture analysis of the two carotid plaques. Noted the radiation-induced carotid plaque has more calcification $(\mathrm{C})$ than the non-radiation-induced carotid plaque (D). DPTA, detailed plaque texture analysis; RT, radiotherapy; NPC, nasopharyngeal carcinoma; CCA, common carotid artery; CVRF, cardiovascular risk factor.

existed studies that found the increased CIMT as an early alteration after irradiation, when compared to the carotid artery without irradiation $(25,26)$. However, with respect to the comparison of radiation-induced atherosclerosis and spontaneous atherosclerosis, this finding was inconsistent with the results of the previous study. The discrepancy of the two studies may mainly arise from different study subjects. Previous study demonstrated the comparison of CIMT between post-RT patients ( $\geq 4$ yrs) and type- 2 diabetics, while our study compared CIMT value of postRT patients ( $\geq 4 \mathrm{yrs}$ ) and subjects commonly with multiple CVRFs. More CVRFs exerted cumulative stress on vessel wall, and thus led to a greater CIMT.

Moreover, in post-RT patients, CIMT was noted to have significant association with previous history of radiation therapy, patient's age, and number of CVRF by present study. The CIMT increased with the previous irradiation, advancing age and more CVRFs. Result of the present study was consistent with previous studies which found that radiation, patient's age and the presence of CVRF played an important role in carotid artery wall thickening (27-31).

The radiation-induced carotid artery wall thickening could be explained from two aspects. The first one is the direct injury to endothelium, resulting in a series of biological responses of endothelial cells to ionizing radiation. In patients with previous irradiation, CIMT was the manifestation of endothelial impairment caused by ionizing radiation. Exposure to the ionizing radiation caused double strands DNA damage of endothelial cells. When the rate of DNA repair was not high enough to suppress the damage, the accumulated injury induced the expression of adhesion molecules, and thereby promoted the atherosclerosis by facilitating the circulating immune cells anchoring on the endothelium. This eventually resulted in the advanced atherosclerosis through inflammation, cell apoptosis, proliferation and fibrosis 
Table 7 Effects of various risk factors on plaque characteristics by multivariate analysis, with the adjustment of age and sex

\begin{tabular}{|c|c|c|c|c|c|c|c|c|c|c|}
\hline Risk factors & \multicolumn{3}{|c|}{ CIMT } & \multicolumn{3}{|c|}{ Plaque formaXtion } & \multicolumn{3}{|c|}{ Number of plaque per subject } & $\begin{array}{c}\text { Overall } \mathrm{P} \\
\text { value }\end{array}$ \\
\hline Age & $0.181[1,210]$ & 46.510 & $<0.001^{\star * *}$ & $0.136[1,210]$ & 13.442 & $<0.001^{\star \star \star}$ & $0.060[1,210]$ & 33.090 & $<0.001^{\star \star \star}$ & $<0.001^{\star * \star}$ \\
\hline Sex & $0.008[1,210]$ & 1.717 & 0.192 & $0.004[1,210]$ & 0.935 & 0.335 & $0.006[1,210]$ & 1.341 & 0.248 & 0.346 \\
\hline Number of CVRF & $0.029[2,210]$ & 3.018 & $0.048^{*}$ & $0.006[2,210]$ & 0.638 & 0.529 & $0.024[2,210]$ & 2.616 & 0.075 & $0.025^{\star}$ \\
\hline
\end{tabular}

Number of CVRFs were grouped by subjects without CVRF, with one CVRF or at least two CVRFs. ${ }^{*}, \mathrm{P}<0.05 ;{ }^{* *}, \mathrm{P}<0.01 ;{ }^{* \star *}, \mathrm{P}<0.001$.

CIMT, carotid intima-media thickness; CVRF, cardiovascular risk factor.

Table 8 Effects of various risk factors on plaque components characteristics by multivariate analysis

\begin{tabular}{|c|c|c|c|c|c|c|c|c|c|c|c|c|}
\hline Risk factors & \multicolumn{3}{|c|}{ Area percentage-lipid } & \multicolumn{3}{|c|}{ Area percentage-calcification } & \multicolumn{3}{|c|}{ IOD-muscle } & \multicolumn{3}{|c|}{ IOD-calcification } \\
\hline Age & 0.063 & 20.121 & $<0.001^{\star \star \star}$ & 0.056 & 17.802 & $<0.001^{\star \star \star}$ & 0.002 & 0.625 & 0.430 & 0.011 & 3.448 & 0.064 \\
\hline Sex & 0.001 & 0.156 & 0.694 & 0.003 & 0.875 & 0.350 & 0.004 & 1.280 & 0.259 & 0.006 & 1.728 & 0.190 \\
\hline Number of CVRF & 0.006 & 0.855 & 0.426 & 0.005 & 0.717 & 0.489 & 0.014 & 2.109 & 0.123 & 0.013 & 1.955 & 0.143 \\
\hline
\end{tabular}

Number of CVRFs were grouped by subjects without CVRF, with one CVRF or $\geq$ two CVRFs. ${ }^{*}, \mathrm{P}<0.05$; ${ }^{* * *}, \mathrm{P}<0.001$. CVRF, cardiovascular risk factor.

in the artery wall $(13,32)$. Subsequently, the intima was thickened because of the deposition of lipids, and the accumulation of macrophages, smooth muscle cells and extracellular matrix. The second one is the impairment of vasa vasorum caused by radiation. Vasa vasorum was microvascular network and vulnerable to radiation. Elevated vascular oxidative stress due to diabetes and hypertension induced posttranslational oxidative modification of proteins, resulting cellular damage and vascular dysfunction, which initiated the early atherosclerosis. CVRF (such as hypertension) could also cause the hypertrophy and hyperplasia of SMC, as well as increase density of collagen and elastin in media of an artery (33). These events eventually resulted in a thickening of CIMT.

\section{Carotid plaque formation}

Given that plaque formation is a consequence of carotid atherosclerosis, the presence of carotid plaque was a useful indicator to monitor the progression of carotid atherosclerosis and predict cerebrovascular disease. Some studies found post-RT head and neck cancer patients were more likely to suffer from stroke/transient ischemic attack
(TIA) (34-36). In a mean of 9.5 years follow-up study, with 6,700 participates and $>500$ cardiovascular disease events, carotid plaque formation was found to be a strong predictor of stroke/TIA in asymptomatic subjects $(\mathrm{P}=0.045)$, compared with coronary artery calcium $(\mathrm{P}=0.438)$ and high CIMT ( $\mathrm{P}=0.160)$ (37). Therefore, the combined assessments of CIMT and carotid plaque might provide more comprehensive assessment of radiation-induced carotid atherosclerosis for early detection of carotid atherosclerosis and better predicting the future cerebrovascular disease.

In this study, result showed that more carotid plaques were formed in radiation-induced carotid atherosclerosis when compared to non-radiation-induced carotid atherosclerosis, and the number of carotid plaques in post-RT NPC patients increased with advancing age. Besides, radiation-induced carotid atherosclerosis, regardless of the presence of CVRFs, had a higher carotid plaque formation than non-radiationinduced carotid atherosclerosis. The finding was consistent with that of previous study, which found that post-RT NPC patients had more carotid plaques than the health, and higher plaque burden than DM patients (13).

Although the pathogenesis of radiation-induced carotid plaque has not been fully revealed, the carotid plaque 
formation caused by radiation was a consequence of the combination of direct vascular injury, inflammatory and accelerated atherosclerosis $(25,38,39)$. Endothelial dysfunction was the primary response to radiation eliciting direct injury on vascular. Endothelium barrier was destructed and permitted oxidative-LDL accumulation in the sub-endothelial space, as well as migration of circulating lymphocytes and monocytes to intima. Monocytes derived to macrophages scavenging lipids and formed foam cells. Meanwhile, SMCs migrated to intima and proliferated in media, which caused by platelet-derived growth factor and basic fibroblast growth factor released by platelets. The accumulation of lymphocytes, macrophages, foam cells and SMCs formed an early lesion of carotid artery (40).

Accelerated atherosclerosis was promoted by inflammation and endothelial-to-mesenchymal transition.

Thus, to assess carotid plaque vulnerability, GSM is more commonly used in conjunction with other parameters such as degree of carotid artery stenosis and CIMT (28,41-43). A previous study demonstrated that there was no significant difference in GSM between radiation-induced and nonradiation-induced carotid plaques (28). Result of the present study was consistent with the previous study. We found that the GSM of radiation-induced and non-radiation-induced carotid plaques were not significantly different. To further evaluate the difference of these two types of carotid plaque in detail, a DPTA of carotid plaques was conducted in the present study.

Numerous studies have reported the application of different medical imaging techniques in carotid plaque component analysis. Magnetic resonance imaging (MRI) is a robust imaging tool for soft tissue examination and has been used for the evaluation of carotid plaque composition (44-46). Intravascular ultrasound (IVUS) elastography $(47,48)$ and acoustic radiation force impulse (ARFI) elastography ultrasound $(49,50)$ can be used to estimate the carotid plaque stiffness and assess the plaque composition. Multi-detector computed tomography angiography (CTA) can assess carotid plaque complicated with intraplaque haemorrhage with high sensitivity (100\%) and inter-observer reliability (64.7\%) (51). Meanwhile, some drawbacks should be aware when using these imaging modalities for carotid plaque assessment. MRI is not suitable for patients with metal implants or pacemakers. MRI examination is costly and time-consuming. IVUS is an invasive procedure and the clinical value of ARFI elastography for investigation of carotid plaque composition is uncertain due to the unavailability of comprehensive study. CTA cannot distinguish intra-plaque haemorrhage and lipids, and the examination involves ionizing radiation and administration of iodinated contrast agent which is not suitable for patients with deteriorated renal function (52).

In the DPTA of carotid plaques in the present study, there were more radiation-induced plaques contained calcification than non-radiation-induced plaques. Radiationinduced carotid plaque tended to be more hyperechoic. The results are consistent with a previous study which found that calcified carotid plaques were common in post-RT head and neck cancer patients (26).

The causes of radiation-induced atherosclerosis with more calcification in carotid plaques may be resulted from the serial responses of mesenchymal stem cells (MSCs) after ionizing radiation. Under the process of atherosclerosis, the existed osteo-like cells could induce MSC differentiation, and the MSCs would differentiate into calcified vascular smooth muscle cells, which facilitate the progress of vascular calcification (53). Although the conditioned medium of MSCs (medium harvest from cultured MSCs) plays the protective role by anti-inflammation, anti-apoptosis, regulating the Wnt signal in a negative way and inhibiting the combination of BMP2 signal, the excessive oxidative stress could still trigger the amplified atherosclerosis responses. Moreover, previous studies found the progression of radiation-induced carotid atherosclerosis was involved in caspase-mediated apoptosis $(54,55)$ and excessive production of reactive oxidative species (ROS) $(54,56,57)$. Results of these previous studies demonstrated that lipid peroxidation was more likely to be triggered in the radiation-induced atherosclerotic lesion with abundant of ROS. Therefore, lipids peroxidation may account for the lesser lipid deposition within the plaques in radiation-induced carotid atherosclerosis.

\section{Limitations}

The sample size of the two patient/subject group was small, further studies with a larger sample size are suggested. In the study, we only used one image analysis method, i.e., DPTA, to evaluate the plaque texture. However, newer image analysis methods for carotid plaque texture evaluation and new plaque texture features for predicting risk of stroke are available $(58,59)$.

\section{Conclusions}

Radiation caused more severe carotid artery disease than CVRF with larger CIMT and more prevalent of carotid 
plaque. The presence or absence of CVRF in post-RT NPC patients might not affect the degree of carotid atherosclerosis. Radiation-induced carotid plaques tended to have more intra-plaque calcifications, whereas nonradiation-induced carotid plaques seemed to have more lipids. Ultrasound aided by computer-assisted image analysis has potential for more accurate assessment of carotid atherosclerosis.

\section{Acknowledgments}

We thank the assistance of the staff in the Department of Clinical Oncology, Queen Mary Hospital.

Funding: This work was supported by a research studentship (RUAS) and a research grant (P0008624, UAB9) of the Hong Kong Polytechnic University.

\section{Footnote}

Conflicts of Interest: All authors have completed the ICMJE uniform disclosure form (available at http://dx.doi. org/10.21037/qims-20-1012). The authors have no conflicts of interest to declare.

Ethical Statement: The Human Subject Ethics Subcommittee of the Hong Kong Polytechnic University (HSEARS20160930001) and the Institutional Review Board of the University of Hong Kong/Hospital Authority Hong Kong West Cluster approved this study (HA RE001F3). All participants (recruited post-RT NPC patients and CVRF subjects) were provided an information sheet with detailed information of the study and the rights of participants. Informed written consent was obtained from all participants before the commencement of the examinations.

Open Access Statement: This is an Open Access article distributed in accordance with the Creative Commons Attribution-NonCommercial-NoDerivs 4.0 International License (CC BY-NC-ND 4.0), which permits the noncommercial replication and distribution of the article with the strict proviso that no changes or edits are made and the original work is properly cited (including links to both the formal publication through the relevant DOI and the license). See: https://creativecommons.org/licenses/by-nc-nd/4.0/.

\section{References}

1. Bray F, Ferlay J, Soerjomataram I, Siegel RL, Torre LA,
Jemal A. Global cancer statistics 2018: GLOBOCAN estimates of incidence and mortality worldwide for 36 cancers in 185 countries. CA Cancer J Clin 2018;68:394-424.

2. Chong VF, Ong CK. Nasopharyngeal carcinoma. Eur J Radiol 2008;66:437-47.

3. Lee AW, Ma BB, Ng WT, Chan AT. Management of nasopharyngeal carcinoma: current practice and future perspective. J Clin Oncol 2015;33:3356-64.

4. Leung TW, Tung SY, Sze WK, Wong FC, Yuen KK, Lui CM, Lo SH, Ng TY, O SK. Treatment results of 1070 patients with nasopharyngeal carcinoma: an analysis of survival and failure patterns. Head Neck 2005;27:555-65.

5. Glastonbury CM. Nasopharyngeal carcinoma: the role of magnetic resonance imaging in diagnosis, staging, treatment, and follow-up. Top Magn Reson Imaging 2007;18:225-35.

6. Naqvi TZ, Lee MS. Carotid intima-media thickness and plaque in cardiovascular risk assessment. JACC Cardiovasc Imaging 2014;7:1025-38.

7. Touboul PJ, Hernandez-Hernandez R, Kucukoglu S, Woo KS, Vicaut E, Labreuche J, Migom C, Silva H, Vinueza R, Investigators P-A. Carotid artery intima media thickness, plaque and Framingham cardiovascular score in Asia, Africa/Middle East and Latin America: the PARC-AALA study. Int J Cardiovasc Imaging 2007;23:557-67.

8. Stein JH, Korcarz CE, Hurst RT, Lonn E, Kendall CB, Mohler ER, Najjar SS, Rembold CM, Post WS, American Society of Echocardiography Carotid IntimaMedia Thickness Task Force. Use of carotid ultrasound to identify subclinical vascular disease and evaluate cardiovascular disease risk: a consensus statement from the American Society of Echocardiography Carotid IntimaMedia Thickness Task Force. Endorsed by the Society for Vascular Medicine. J Am Soc Echocardiogr 2008;21:93111; quiz 89-90.

9. Ross R. Atherosclerosis--an inflammatory disease. N Engl J Med 1999;340:115-26.

10. Lusis AJ. Atherosclerosis. Nature 2000;407:233-41.

11. Sumpio BE, Riley JT, Dardik A. Cells in focus: endothelial cell. Int J Biochem Cell Biol 2002;34:1508-12.

12. Lam WW, Leung SF, So NM, Wong KS, Liu KH, Ku PK, Yuen HY, Metreweli C. Incidence of carotid stenosis in nasopharyngeal carcinoma patients after radiotherapy. Cancer 2001;92:2357-63.

13. Yuan C, Wu VW, Yip SP, Kwong DL, Ying M. Ultrasound evaluation of carotid atherosclerosis in post-radiotherapy nasopharyngeal carcinoma patients, type 2 diabetics, and 
healthy controls. Ultraschall Med 2017;38:190-7.

14. Lal BK, Hobson RW 2nd, Pappas PJ, Kubicka R, Hameed M, Chakhtoura EY, Jamil Z, Padberg FT Jr, Haser PB, Duran WN. Pixel distribution analysis of B-mode ultrasound scan images predicts histologic features of atherosclerotic carotid plaques. J Vasc Surg 2002;35:1210-7.

15. Madycki G, Staszkiewicz W, Gabrusiewicz A. Carotid plaque texture analysis can predict the incidence of silent brain infarcts among patients undergoing carotid endarterectomy. Eur J Vasc Endovasc Surg 2006;31:373-80.

16. Yuan C, Wu VW, Yip SP, Kwong DL, Ying M. Predictors of the extent of carotid atherosclerosis in patients treated with radiotherapy for nasopharyngeal carcinoma. PLoS One 2014;9:e116284.

17. Touboul PJ, Hennerici MG, Meairs S, Adams H, Amarenco P, Bornstein N, Csiba L, Desvarieux M, Ebrahim S, Hernandez Hernandez R, Jaff M, Kownator S, Naqvi T, Prati P, Rundek T, Sitzer M, Schminke U, Tardif JC, Taylor A, Vicaut E, Woo KS. Mannheim carotid intima-media thickness and plaque consensus (2004-20062011). An update on behalf of the advisory board of the $3 \mathrm{rd}$, 4th and 5 th watching the risk symposia, at the 13 th, 15 th and 20th European Stroke Conferences, Mannheim, Germany, 2004, Brussels, Belgium, 2006, and Hamburg, Germany, 2011. Cerebrovasc Dis 2012;34:290-6.

18. Pedro LM, Pedro MM, Goncalves I, Carneiro TF, Balsinha C, Fernandes e Fernandes R, Fernandes e Fernandes J. Computer-assisted carotid plaque analysis: characteristics of plaques associated with cerebrovascular symptoms and cerebral infarction. Eur J Vasc Endovasc Surg 2000;19:118-23.

19. Tegos TJ, Sabetai MM, Nicolaides AN, Pare G, Elatrozy TS, Dhanjil S, Griffin M. Comparability of the ultrasonic tissue characteristics of carotid plaques. J Ultrasound Med 2000;19:399-407.

20. Grønholdt ML, Nordestgaard BG, Bentzon J, Wiebe BM, Zhou J, Falk E, Sillesen H. Macrophages are associated with lipid-rich carotid artery plaques, echolucency on B-mode imaging, and elevated plasma lipid levels. J Vasc Surg 2002;35:137-45.

21. Bassiouny HS, Sakaguchi Y, Mikucki SA, McKinsey JF, Piano G, Gewertz BL, Glagov S. Juxtalumenal location of plaque necrosis and neoformation in symptomatic carotid stenosis. J Vasc Surg 1997;26:585-94.

22. Pignoli P, Tremoli E, Poli A, Oreste P, Paoletti R. Intimal plus medial thickness of the arterial wall: a direct measurement with ultrasound imaging. Circulation
1986;74:1399-406.

23. Wendelhag I, Gustavsson T, Suurkula M, Berglund G, Wikstrand J. Ultrasound measurement of wall thickness in the carotid artery: fundamental principles and description of a computerized analysing system. Clin Physiol 1991;11:565-77.

24. Wikstrand J. Methodological considerations of ultrasound measurement of carotid artery intima-media thickness and lumen diameter. Clin Physiol Funct Imaging 2007;27:341-5.

25. Fernández-Alvarez V, López F, Suárez C, Strojan P, Eisbruch A, Silver CE, Mendenhall WM, Langendijk JA, Rinaldo A, Lee AWM, Beitler JJ, Smee R, Alvarez J, Ferlito A. Radiation-induced carotid artery lesions. Strahlenther Onkol 2018;194:699-710.

26. Toprak U, Aytas I, Ustuner E, Habiboglu R, Aslan N, Pasaoglu E, Karademir A. Sonographic assessment of acute changes in plaque size and echogenicity and in intimamedia thickness of carotid arteries after neck radiation therapy. J Clin Ultrasound 2012;40:566-71.

27. Gujral DM, Shah BN, Chahal NS, Bhattacharyya S, Hooper J, Senior R, Harrington KJ, Nutting CM. Carotid intima-medial thickness as a marker of radiation-induced carotid atherosclerosis. Radiother Oncol 2016;118:323-9.

28. Cheng SW, Ting AC, Wu LL. Ultrasonic analysis of plaque characteristics and intimal-medial thickness in radiation-induced atherosclerotic carotid arteries. Eur J Vasc Endovasc Surg 2002;24:499-504.

29. Bilora F, Pietrogrande F, Petrobelli F, Polato G, Pomerri $\mathrm{F}$, Muzzio PC. Is radiation a risk factor for atherosclerosis? An echo-color Doppler study on Hodgkin and nonHodgkin patients. Tumori 2006;92:295-8.

30. de Freitas EV, Brandao AA, Pozzan R, Magalhies ME, Castier M, Brandao AP. Study of the intima-media thickening in carotid arteries of healthy elderly with high blood pressure and elderly with high blood pressure and dyslipidemia. Clin Interv Aging 2008;3:525-34.

31. Kruglikova AS, Strazhesko ID, Plokhova EV, Pyhtina VS, Akasheva DU, Isaykina OU, Tkacheva ON, Boytsov SA. P3.10 Genetic factors vs cardiovascular risk factors. What is more significant in vascular aging? Artery Research 2013;7:abstr 129.

32. Gray K, Bennett M. Role of DNA damage in atherosclerosis--bystander or participant? Biochem Pharmacol 2011;82:693-700.

33. Michel JB, Tedgui A, Salzmann JL, Belmain J, Levy B. Function and structure of the vascular wall in experimental arterial hypertension. Effect of treatment. Nephrologie 
1989;10:55-7.

34. Dorresteijn LD, Kappelle AC, Boogerd W, Klokman WJ, Balm AJ, Keus RB, van Leeuwen FE, Bartelink H. Increased risk of ischemic stroke after radiotherapy on the neck in patients younger than 60 years. J Clin Oncol 2002;20:282-8.

35. Huang YS, Lee CC, Chang TS, Ho HC, Su YC, Hung SK, Lee MS, Chou P, Chang YH, Lee CC. Increased risk of stroke in young head and neck cancer patients treated with radiotherapy or chemotherapy. Oral Oncol 2011;47:1092-7.

36. Gujral DM, Chahal N, Senior R, Harrington KJ, Nutting CM. Radiation-induced carotid artery atherosclerosis. Radiother Oncol 2014;110:31-8.

37. Gepner AD, Young R, Delaney JA, Tattersall MC, Blaha MJ, Post WS, Gottesman RF, Kronmal R, Budoff MJ, Burke GL, Folsom AR, Liu K, Kaufman J, Stein JH. Comparison of coronary artery calcium presence, carotid plaque presence, and carotid intima-media thickness for cardiovascular disease prediction in the Multi-Ethnic Study of Atherosclerosis. Circ Cardiovasc Imaging 2015;8:e002262.

38. Stewart FA, Heeneman S, Te Poele J, Kruse J, Russell NS, Gijbels M, Daemen M. Ionizing radiation accelerates the development of atherosclerotic lesions in ApoE-/- mice and predisposes to an inflammatory plaque phenotype prone to hemorrhage. Am J Pathol 2006;168:649-58.

39. Tribble DL, Barcellos-Hoff MH, Chu BM, Gong EL. Ionizing radiation accelerates aortic lesion formation in fat-fed mice via SOD-inhibitable processes. Arterioscler Thromb Vasc Biol 1999;19:1387-92.

40. Gujral DM, Shah BN, Chahal NS, Senior R, Harrington KJ, Nutting CM. Clinical features of radiation-induced carotid atherosclerosis. Clin Oncol (R Coll Radiol) 2014;26:94-102.

41. Biasi GM, Sampaolo A, Mingazzini P, De Amicis P, ElBarghouty N, Nicolaides AN. Computer analysis of ultrasonic plaque echolucency in identifying high risk carotid bifurcation lesions. Eur J Vasc Endovasc Surg 1999;17:476-9.

42. Ibrahimi P, Jashari F, Johansson E, Gronlund C, Bajraktari G, Wester P, Henein MY. Common carotid intimamedia features determine distal disease phenotype and vulnerability in asymptomatic patients. Int J Cardiol 2015;196:22-8.

43. Martínez-Sánchez P, Fernández-Domínguez J, Ruiz-Ares G, Fuentes B, Alexandrov AV, Díez-Tejedor E. Changes in carotid plaque echogenicity with time since the stroke onset: an early marker of plaque remodeling? Ultrasound Med Biol 2012;38:231-7.

44. Kerwin WS, O'Brien KD, Ferguson MS, Polissar N, Hatsukami TS, Yuan C. Inflammation in carotid atherosclerotic plaque: a dynamic contrast-enhanced MR imaging study. Radiology 2006;241:459-68.

45. Sun J, Canton G, Balu N, Hippe DS, Xu D, Liu J, Hatsukami TS, Yuan C. Blood pressure is a major modifiable risk factor implicated in pathogenesis of intraplaque hemorrhage: an in vivo magnetic resonance imaging study. Arterioscler Thromb Vasc Biol 2016;36:743-9.

46. Lin R, Chen S, Liu G, Xue Y, Zhao X. Association between carotid atherosclerotic plaque calcification and intraplaque hemorrhage: a magnetic resonance imaging study. Arterioscler Thromb Vasc Biol 2017;37:1228-33.

47. de Korte CL, Pasterkamp G, van der Steen AF, Woutman HA, Bom N. Characterization of plaque components with intravascular ultrasound elastography in human femoral and coronary arteries in vitro. Circulation 2000;102:617-23.

48. de Korte CL, van der Steen AF, Cepedes EI, Pasterkamp G, Carlier SG, Mastik F, Schoneveld AH, Serruys PW, Bom N. Characterization of plaque components and vulnerability with intravascular ultrasound elastography. Phys Med Biol 2000;45:1465-75.

49. Allen JD, Ham KL, Dumont DM, Sileshi B, Trahey GE, Dahl JJ. The development and potential of acoustic radiation force impulse (ARFI) imaging for carotid artery plaque characterization. Vasc Med 2011;16:302-11.

50. Czernuszewicz TJ, Homeister JW, Caughey MC, Farber MA, Fulton JJ, Ford PF, Marston WA, Vallabhaneni R, Nichols TC, Gallippi CM. Non-invasive in vivo characterization of human carotid plaques with acoustic radiation force impulse ultrasound: comparison with histology after endarterectomy. Ultrasound Med Biol 2015;41:685-97.

51. Ajduk M, Pavic L, Bulimbasic S, Sarlija M, Pavic P, Patrlj L, Brkljacic B. Multidetector-row computed tomography in evaluation of atherosclerotic carotid plaques complicated with intraplaque hemorrhage. Ann Vasc Surg 2009;23:186-93.

52. Naim C, Douziech M, Therasse E, Robillard P, Giroux MF, Arsenault F, Cloutier G, Soulez G. Vulnerable atherosclerotic carotid plaque evaluation by ultrasound, computed tomography angiography, and magnetic resonance imaging: an overview. Can Assoc Radiol J 2014;65:275-86.

53. Xie C, Ouyang L, Chen J, Zhang H, Luo P, Wang J, 
Huang H. The emerging role of mesenchymal stem cells in vascular calcification. Stem Cells Int 2019;2019:2875189.

54. Winn RK, Harlan JM. The role of endothelial cell apoptosis in inflammatory and immune diseases. J Thromb Haemost 2005;3:1815-24.

55. Tricot O, Mallat Z, Heymes C, Belmin J, Leseche G, Tedgui A. Relation between endothelial cell apoptosis and blood flow direction in human atherosclerotic plaques. Circulation 2000;101:2450-3.

56. Azzam EI, Jay-Gerin JP, Pain D. Ionizing radiationinduced metabolic oxidative stress and prolonged cell

Cite this article as: $\mathrm{Li} \mathrm{Y,} \mathrm{Kwong} \mathrm{DLW,} \mathrm{Wu} \mathrm{VWC,} \mathrm{Yip} \mathrm{SP,}$ Law HKW, Lee SWY, Ying MTC. Computer-assisted ultrasound assessment of plaque characteristics in radiationinduced and non-radiation-induced carotid atherosclerosis. Quant Imaging Med Surg 2021;11(6):2292-2306. doi: 10.21037/ qims-20-1012 injury. Cancer Lett 2012;327:48-60.

57. Kim W, Lee S, Seo D, Kim D, Kim K, Kim E, Kang J, Seong KM, Youn H, Youn B. Cellular stress responses in radiotherapy. Cells 2019;8:1105.

58. van Engelen A, Wannarong T, Parraga G, Niessen WJ, Fenster A, Spence JD, de Bruijne M. Three-dimensional carotid ultrasound plaque texture predicts vascular events. Stroke 2014;45:2695-701.

59. Awad J, Krasinski A, Parraga G, Fenster A. Texture analysis of carotid artery atherosclerosis from three-dimensional ultrasound images. Med Phys 2010;37:1382-91. 